\title{
Enantema vesicular palatofaríngeo, hallazgo temprano de COVID-19
}

\author{
Palatopharyngeal vesicular enanthem, early finding of COVID-19 \\ José Domínguez-Rodas ${ }^{1}$, Sergio A. Ramírez-García², Nory O. Dávalos-Rodríguez ${ }^{3}$ y \\ Carlos E. Cabrera-Pivaral * \\ ${ }^{1}$ Servicios Médicos Profesionales, A.C. Miahuatlán de Porfirio Díaz, Oaxaca; ${ }^{2}$ Universidad de la Sierra Sur, SUNEO, Miahuatlán de Porfirio Díaz, \\ Oaxaca; ${ }^{3}$ Departamento de Biología Molecular y Genómica, Centro Universitario de Ciencias de la Salud (CUCS), Universidad de Guadalajara, \\ Jalisco; ${ }^{4}$ Departamento de Salud Pública, CUCS, Universidad de Guadalajara, Jalisco. México
}

\begin{abstract}
Resumen
El diagnóstico temprano de la infección por SARS-CoV-2 es muy importante para establecer un tratamiento oportuno. En el presente reporte, en la exploración realizada en otorrinolaringología encontramos un enantema vesicular aperlado en el paladar superior en 954 de 958 pacientes con la cepa clásica, y no se encontró en pacientes con la cepa inglesa. Este hallazgo no se había reportado. Los pacientes fueron tratados a tiempo exitosamente y solo dos pacientes murieron, lo cual se asoció a diabetes mellitus descompensada. Este reporte sugiere que el enantema vesicular encontrado es patognomónico de COVID-19 por cepas clásicas.
\end{abstract}

Palabras clave: Coronavirus. Exantema. Enantema.

\begin{abstract}
Early diagnosis of SARS-CoV-2 infection is very important to establish timely treatment. In the present report, through the examination carried out in otorhinolaryngology, we found a pearlescent vesicular enanthema in the upper palate in 954/958 patients with the classic strain and it was not found in patients with the English strain. This finding had not been reported. The patients were successfully treated on time, only two patients died, which was associated with decompensated diabetes mellitus. The present report suggests that the vesicular enanthem found is pathognomonic for Covid-19 classic strains.
\end{abstract}

Key words: Coronavirus. Rash. Enanthema.

Sr. Editor:

La otorrinolaringología ofrece una alternativa en el diagnóstico temprano de COVID-19. En una serie de 958 pacientes de COVID-19 confirmados por reacción en cadena de la polimerasa (382 diabéticos) que hemos atendido en la Sierra Sur de Oaxaca se detectó en 954 de ellos un enantema vesicular blanco aperlado en el paladar superior, la orofaringe y la cavidad oral, el cual se presenta en los primeros 3 días de la infección con pocas lesiones, progresa y desaparece cuando la viremia está tratada (Fig. 1). Su evolución es igual en los pacientes diabéticos. No

\section{Correspondencia:}

${ }^{*}$ Carlos E. Cabrera-Pivaral

Sierra Mojada 950, Edificio P

Col. Independencia

Fecha de recepción: 26-03-2021

C.P. 44340 , Guadalajara, Jal., México

E-mail: cabrerapivaral @ prodigy.net.mx
Cir Cir. 2021;89(5):692-693 Contents available at PubMed www.cirugiaycirujanos.com 0009-7411/@ 2021 Academia Mexicana de Cirugía. Publicado por Permanyer. Este es un artículo open access bajo la licencia CC BY-NC-ND (http://creativecommons.org/licenses/by-nc-nd/4.0/). 

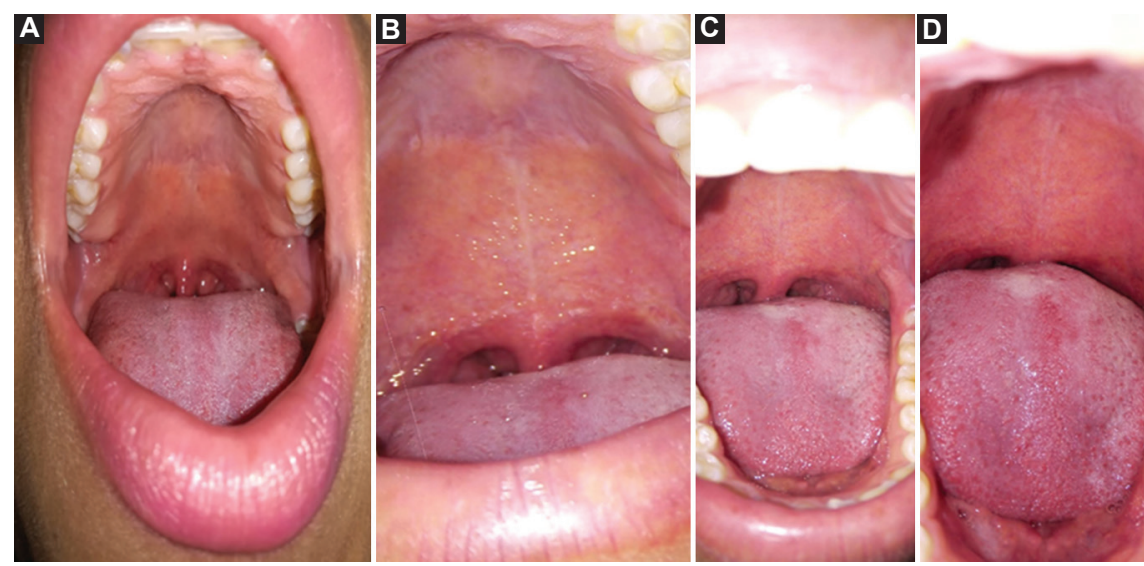

Figura 1. Enantema vesicular palatofaríngeo y su evolución en pacientes con COVID-19. A: primeros tres días de la infección. B: después del tercer día. C: a los 14 días. D: a los 27 días, ya tratada la viremia.

se detectó en cuatro pacientes con la cepa inglesa. Adicionalmente se encontraron la mucosa faríngea y el paladar superior con palidez, áreas amarillas y telangiectasias. No se detectaron eritema, enantema macular ni petequial, maculopetequial o eritematovesicular, lesiones herpetiformes ni úlceras en la lengua, descritos previamente en otros estudios durante los primeros 2-24 días de la enfermedad ${ }^{1-3}$. El enantema presentó una asociación con $\mathrm{X}^{2}$ corregida de 23.18 ( $p=0.0000015$; odds ratio: 213.4 ; intervalo de confianza: 4.1917-926.4). Los pacientes fueron tratados exitosamente con dexametasona, azitromicina, ivermectina y ciclosporina A. Solo dos murieron por diabetes descompensada.

Este reporte sugiere que el enantema encontrado es patognomónico de COVID-19 por cepas clásicas, y se puede considerar una alternativa clínica para el diagnóstico y el tratamiento tempranos; sin embargo, requiere ser correlacionado en posteriores estudios de epidemiología genómica ${ }^{4,5}$.

\section{Agradecimientos}

Los autores agradece a la Fundación Mexicana de Enfermedades Genéticas y Medicina Genómica, AC.

\section{Financiamiento}

Servicios Médicos Profesionales, A.C, Miahuatlán de Porfirio Díaz, Oaxaca, México.

\section{Conflicto de intereses}

Los autores declaran no tener conflictos de intereses.

\section{Responsabilidades éticas}

Protección de personas y animales. Los autores declaran que para esta investigación no se han realizado experimentos en seres humanos ni en animales.

Confidencialidad de los datos. Los autores declaran que han seguido los protocolos de su centro de trabajo sobre la publicación de datos de pacientes.

Derecho a la privacidad y consentimiento informado. Los autores han obtenido el consentimiento informado de los pacientes y/o sujetos referidos en el artículo. Este documento obra en poder del autor de correspondencia.

\section{Bibliografía}

1. Recalcati S. Cutaneous manifestations in COVID-19: a first perspective. J Eur Acad Dermatol Venereol. 2020;34:e212-3.

2. Ornejo OM, Espinoza SI. Covid-19 y manifestaciones orales. Int J Odontostomat. 2020;14:538-9.

3. Jiménez CJ, Ortega QD, de Perosanz LD, Burgos BP, Vañó GS, Fernández GM, et al. Enanthem in patients with COVID-19 and skin rash. JAMA Dermatol. 2020;156:1134-6.

4. Hernández MT, Pérez ML, Romero DC, Martínez CM, Mayoral G, Sánchez NLM, et al. Analysis of SARS-CoV-2 mutations in Mexico, Belize, and isolated regions of Guatemala and its implication in the diagnosis. J Med Virol. 2021;93:2099-114.

5. Taboada $B$, Vázquez JA, Muñoz-Medina JE, Ramos $P$, Escalera M, Boukadida C, et al. Genomic analysis of early SARS-CoV-2 variants introduced in México. J Virol. 2020;94:e01056-20. 